\title{
Topoisomerase II $\alpha$ Gene alteration in Triple Negative Breast Cancer and Its Predictive Role for Anthracycline-Based Chemotherapy (Egyptian NCI Patients)
}

\author{
Mahitab Ibrahim Eltohamy ${ }^{1 *}$, Omnia Mohammed Badawy ${ }^{1}$, Naglaa El kinaai², \\ Iman Loay', Hanan Ramadan Nassar ${ }^{3}$, Rasha Mahmoud Allam ${ }^{4}$, Mona Ali Sakr ${ }^{1}$
}

\begin{abstract}
Objective: Triple negative breast cancer is an aggressive variant of breast cancer; it forms about $15 \%$ of breast cancer cases. It lacks the responsiveness to hormonal and targeted therapies. Anthracyclines remain the treatment option for these patients. Anthracyclines are cardiotoxic, so predicting sensitivity of response by biological predictors may have a role in selecting suitable candidates for these drugs. Material and methods: This study included 50 TNBC cases, from National Cancer Institute, Cairo University(NCI-CU), Egypt, who underwent surgery and received adjuvant chemotherapy. Archived blocks were obtained and immunostaining for Ki-67 LI and Fluorescent In situ Hybridization (FISH) technique to assess TOP2A gene copy number and chromosome 17CEP status were done. Analysis of association between TOP2A alterations and CEP17 polysomy as well as Ki-67 LI with other clinicopathological parameters was done. Associations between the biological markers and event free survival (EFS) and overall survival (OS), were also performed. Results: TOP2A alteration was seen in 9/50 cases ( 5 amplified and 4 deleted). CEP17 Polysomy was detected in $14 \%$ of cases. Most of patients ( $80 \%$ ) showed $\mathrm{Ki}-67 \mathrm{LI} \geq 20 \%$. There was a significant association between TOP2A gene and CEP17 status. Outcome was better with abnormal TOP2A gene status and CEP17 polysomy, radiotherapy and combined anthracyclines and taxanes in the adjuvant setting, however P-values were not significant. Conclusion: TOP2A gene alterations and CEP17 polysomy may have prognostic and predictive role in TNBC treated with adjuvant Anthracyclines.
\end{abstract}

Keywords: TOP2A- CEP17 polysomy-anthracyclines-TNBC

Asian Pac J Cancer Prev, 19 (12), 3581-3589

\section{Introduction}

Breast cancer is the most common cancer among women and it ranks the second cause of cancer deaths in women after lung cancer (Siegel et al., 2018). TNBC accounts for $13.9 \%$ of breast cancer cases in Egyptian population according to (NCI-CU) 12 years cancer registry (Mohamed, 2016).

TNBC is an aggressive variant and lacks the response to hormonal and targeted therapies (Yao et al., 2015). Chemotherapy (specially) Anthracyclines remains the most prominent treatment for these patients (Gianni et al., 2009). The anthracyclines are associated with major toxicity (Almeida et al., 2014), and TNBCs show molecular heterogenicity (Ballinger et al., 2016), so show different sensitivity to the treatment, here came the importance of selecting the appropriate candidates for therapy to avoid exposure to the hazards of unnecessary treatment .
Chromosome 17 is the second most gene-dense chromosome in the human genome, containing many genes involved in breast cancer and DNA repair such as HER-2, TOP2A, BRCA1 and TP53 (Greenberg, 2008) and (Olivier et al., 2009). The region 17q12-q21 contains HER-2 gene, which is responsible for breast cancer progression and poor prognosis. Aberrations in this region predict the response to chemotherapeutic agents (Arriola et al., 2008).

The region 17q21 22 contains the topoisomerase II $\alpha$ gene (Kellner et al., 2002). TOP2A is a nuclear DNA binding enzyme and functions by reducing DNA twisting and supercoiling via cutting both strands of the DNA helix simultaneously, allowing selected regions of the DNA to untangle and engage in transcription, replication and repair processes (Kellner et al., 2002).

TOP2A protein product is the molecular target of anthracycline treatment (Jacot et al., 2013). The gene is amplified in $12-24 \%$ of breast cancers (Fritz et al., 
2005; Knoop et al., 2005) and it is thought to be linked to response to treatment; however, data are conflicting (Jacot et al., 2013).

$\mathrm{Ki}-67$ is a nuclear antigen, expressed in all phases of the cell cycle except G-0 phase of the quiescent cells (Gerdes et al., 1983; Brown and Gatter, 2002). Many studies proved that $\mathrm{Ki}-67$ expression is a useful prognostic factor in breast carcinoma (Yamamoto et al., 2013; Nishimura et al., 2014).

Our aim of study was to assess TOP2A gene alterations and chromosome 17CEP statuses as well as $\mathrm{Ki}-67 \mathrm{LI}$ in cases of TNBC and to analyze their association with other clinicopathological parameters as well as EFS and OS.

\section{Materials and Methods}

This retrospective study was carried on 50 cases diagnosed as TNBC at the (NCI-CU) during the period from January 1st, 2012 till January 1st, 2015. The research was approved by the Institutional Review Board of NCI. Written informed consents were obtained from all patients. All the cases were diagnosed invasive duct carcinoma, with 4 cases showing medullary features in addition.

Personal and clinical data of cases were collected from pathology reports. Data regarding tumor stage, adjuvant chemotherapy regimen and follow up outcome were obtained from medical records. Excluded cases were those of distant metastasizing disease and those who received neoadjuvant therapy. Hematoxylin and Eosin (Hx andE) stained slides were reviewed regarding histologic grade according to guidelines proposed by (Elston and Ellis, 1991). Staging of tumors was carried out according to the American Joint Committee on Cancer (AJCC) TNM staging system of breast cancer, 7th edition (Edge and Compton, 2010). Immunostained slides for all cases were revised for their triple negativity status (ER, PR and HER-2/neu). Paraffin blocks were obtained and four $\mu$ sections were prepared for IHC against Ki-67 LI and $3 \mu$ sections were prepared for FISH analysis of TOP2A gene copy number and CEP17 status.

Immunostaining for assessment of proliferation status (Ki-67 LI): Immunostaining was done on Benchmark XT (Ventana) Autostainer. Instructions of protocols were followed. We have used Ki-67 (Ready to use, mouse monoclonal anti-human antibody, MIB-1 clone, DAKODenmark). The cases with KI-67 LI $\geq 20 \%$ were considered of high" Ki-67 status "as recommended by The 13th St Gallen International Breast Cancer Conference (2013) (Untch et al., 2013).

FISH technique for assessment of TOP2A and CEP17 polysomy

We have used ZytoLight SPEC ERBB2/TOP2A/CEN 17 Triple color probe (PL52) and followed the steps provided by the manufacturer. It is designed for the detection of the HER-2 gene as well as the TOP2A gene and chromosome $17 \alpha$ satellites in formalin-fixed, paraffin-embedded tissue by using FISH technique. Evaluation of the slides was done under Zeiss fluorescence microscopy and slides were analyzed with the $\mathrm{x} 100$ objective. A minimum of 20 non-overlapping tumor cell nuclei with signals for both chromosome and gene were counted in each case; the red signals indicated TOP2A; green signals for HER-2, and aqua signals for the CEP17 gene region. Nuclei were counterstained with DAPI. Two signals from the CEP17 gene region were used as internal control. Zeiss imaging software system was used. Three images were captured for each slide at different filters, but the pictures with green signals of HER-2 gene were omitted to avoid overlapping with aqua signals of the chromosome.

Criteria for assessment of TOP2A and Chromosome 17 CEP status

Cases with $\geq 3$ CEP17 hybridization signals detected in $>30 \%$ of counted nuclei were classified as CEP 17 gain (polysomy) (Perez et al., 2010). We have followed the approved term of low level of gene amplification at ratio $1.5-2.0$, and as high level of amplification at ratio greater than 2 and normal gene copy number when gene to chromosome ratio is less than 1.5, after assessment in four areas of 60 non-overlapping tumor nuclei (Mitrović et al., 2014). TOP $2 \mathrm{~A}$ was considered deleted when ratio was $\leq 0.8$ (Olsen et al., 2004; Leo et al., 2011).

\section{Statistical methods}

Data were analyzed using IBM SPSS advanced statistics (Statistical Package for Social Sciences), version 23 (SPSS Inc., Chicago, IL). Numerical data were described as median, range or mean and standard deviation as appropriate, while qualitative data were described as number and percentage. Chi-square /Fisher's exact tests were used to examine the relation between qualitative variables as appropriate. Survival analysis was done by Kaplan-Meier method. Comparison between two survival curves was done by log rank test. Multivariate analysis was done by Cox regression model to test for independent prognostic effect of statistically significant variables on univariate level with calculating hazard ratio and its $95 \%$ confidence interval. A p-value less than or equal to 0.05 was considered statistically significant. OS was calculated from date of diagnosis till date of death or last follow up. EFS was calculated from date of surgery till date of relapse, metastasis, death or last follow up.

\section{Results}

Clinicopathologic parameters of all studied cases (Table 1)

All studied cases were females. Ages ranged from 29 to 77 years with a mean of 50.18 years. Most of cases were $\leq 50$ years, post-menopausal, of grade II and of stage II. Six cases showed positive family history of breast cancer. All the cases were diagnosed invasive duct carcinoma, with 4 cases showed in addition medullary features. Adjuvant Anthracycline based chemotherapy was given to all cases (4-6) cycles. Thirty-six cases were given weekly Taxane in addition (3-12cycles). Adjuvant radiotherapy was given to 24 cases.

\section{Immunohistochemical results}

Majority of the cases (88\%) showed Ki-67 LI $\geq 20 \%$, 
Table 1. Clinicopathological Data of the Studied Cases

\begin{tabular}{|c|c|c|}
\hline Clinicopathological variable & Frequency & Percentage $\%$ \\
\hline \multicolumn{3}{|l|}{ Age(years) } \\
\hline$\leq 50$ & 30 & 60 \\
\hline$>50$ & 20 & 40 \\
\hline \multicolumn{3}{|l|}{ Menopausal status } \\
\hline Postmenopausal & 30 & 60 \\
\hline Premenopausal & 20 & 40 \\
\hline \multicolumn{3}{|l|}{ Family history } \\
\hline Positive & 6 & 12 \\
\hline Negative & 44 & 88 \\
\hline \multicolumn{3}{|l|}{ Tumor histology } \\
\hline Invasive duct carcinoma & 46 & 92 \\
\hline $\begin{array}{l}\text { Carcinoma with medullary } \\
\text { features }\end{array}$ & 4 & 8 \\
\hline \multicolumn{3}{|l|}{ Tumor grade } \\
\hline Grade 1 & 1 & 2 \\
\hline Grade 2 & 32 & 64 \\
\hline Grade 3 & 17 & 34 \\
\hline \multicolumn{3}{|l|}{ Tumor stage } \\
\hline PT1 & 9 & 18 \\
\hline PT2 & 35 & 70 \\
\hline PT3 & 4 & 8 \\
\hline PT4 & 2 & 4 \\
\hline \multicolumn{3}{|l|}{ Lymph node status } \\
\hline Negative & 18 & 36 \\
\hline Positive & 32 & 64 \\
\hline \multicolumn{3}{|l|}{ Disease stage } \\
\hline Stage I & 3 & 6 \\
\hline Stage II & 30 & 60 \\
\hline Stage III & 17 & 34 \\
\hline \multicolumn{3}{|l|}{ Disease status } \\
\hline Local disease & 22 & 44 \\
\hline Locally advanced & 28 & 56 \\
\hline \multicolumn{3}{|l|}{ Adjuvant chemotherapy } \\
\hline Anthracycline only & 14 & 28 \\
\hline Anthracycline with Taxane & 36 & 72 \\
\hline \multicolumn{3}{|l|}{ Adjuvant radiotherapy } \\
\hline Yes & 24 & 48 \\
\hline No & 26 & 52 \\
\hline
\end{tabular}

while $12 \%$ showed Ki-67 LI $<20 \%$. Although no statistically significant associations could be detected between $\mathrm{Ki}-67 \mathrm{LI}$ and clinico-pathologic parameters as well as TOP2A and CEP17 abnormalities, yet it was observed that all the cases with CEP17 polysomy and all cases with TOP $2 \mathrm{~A}$ alterations are of $\mathrm{Ki}-67 \mathrm{LI} \geq 20 \%$ (Table 2).

FISH results: All the cases showed normal HER-2 gene status. Chromosome 17 CEP polysomy was detected in 7 cases (14\%), rest of cases showed normal chromosome diploid status. TOP2A gene amplification was detected in 5 cases $(10 \%)$, deletion was seen in 4 cases $(8 \%)$.

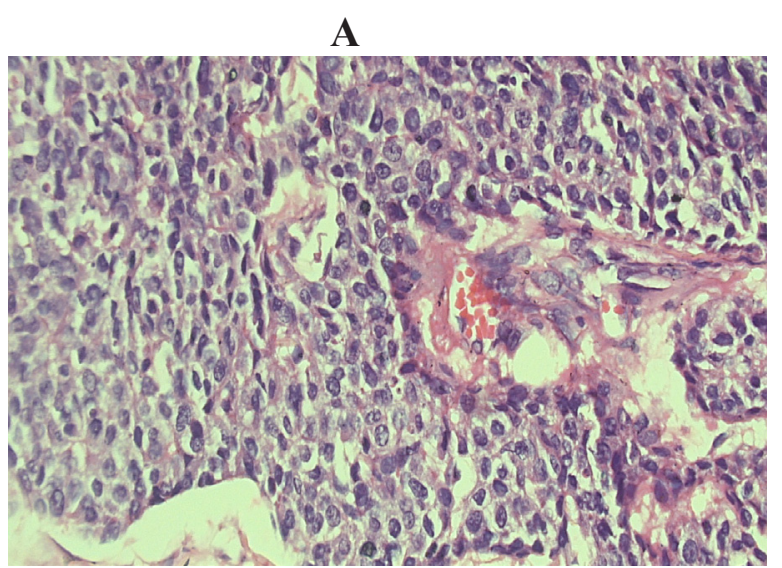

B

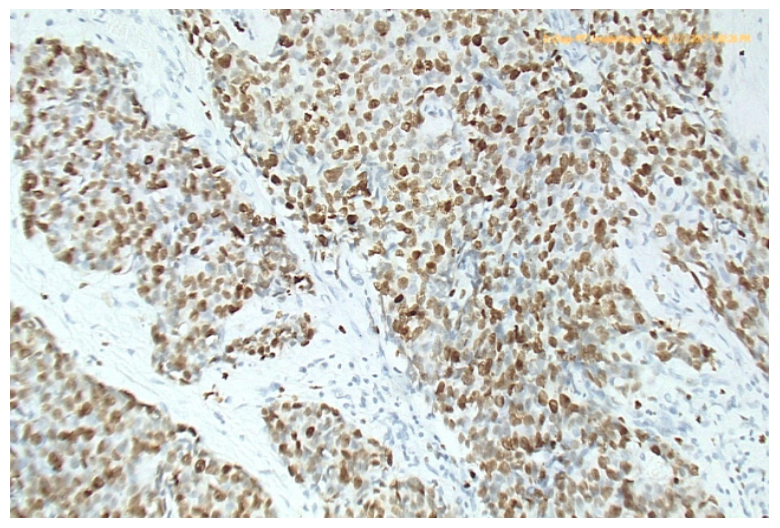

C

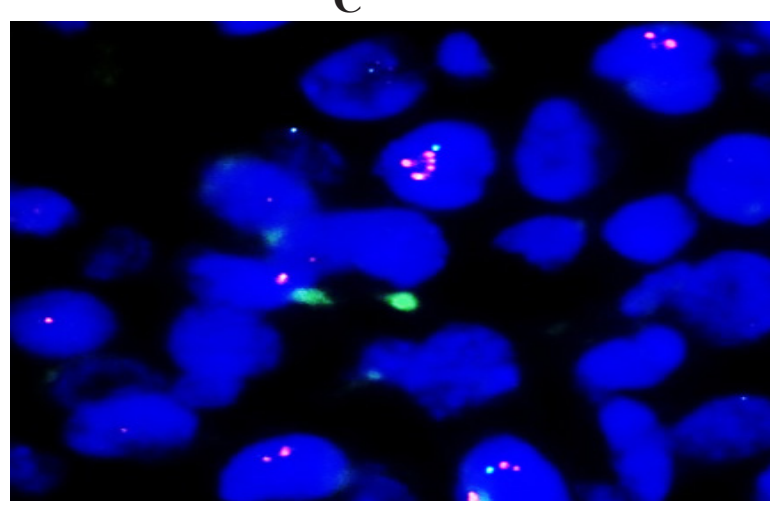

Figure 1. Invasive Duct Carcinoma, Grade III with Normal Chromosome 17 CEP Number and Amplified TOP2A Gene. (A), Histologic section of the tumor, Hx\&E stained (X400); (B), Immunostained slide showing high KI-67 LI about $85 \%(X 200)$; (C), Representative FISH image showing normal CEP17 copy number and amplified TOP2A gene (only 1 Aqua signal per nucleus for the chromosome, due to truncation of nuclei and 2-5 red signals for the gene). Red arrow points to nucleus with amplified TOP2A gene. Gene to chromosome ratio $>2$.

Combined abnormality; chromosome 17 CEP polysomy and TOP2A deletion in the same setting was seen in 4 cases, while combined CEP17 polysomy with TOP2A amplification was seen in 2 cases. Two cases showed TOP2A gene amplification without polysomy and one case showed polysomy with normal TOP2A gene status. There were no statistically significant associations between TOP2A alteration and clinico-pathologic parameters, so as between CEP17 polysomy and clinicopathologic 
Table 2. Correlations between Ki-67 LI and Other Variables

\begin{tabular}{|c|c|c|c|c|}
\hline $\begin{array}{l}\text { Clinicopathological } \\
\text { variable /Category }\end{array}$ & $\begin{array}{c}\mathrm{Ki} 67<20 \% \\
\quad(\mathrm{n}=6)\end{array}$ & $\begin{array}{c}\mathrm{Ki} 67 \geq 20 \% \\
\quad(\mathrm{n}=44)\end{array}$ & $\begin{array}{c}\text { Total } \\
(100 \%) \\
(n=50)\end{array}$ & $\begin{array}{c}\mathrm{P} \\
\text { value }\end{array}$ \\
\hline \multicolumn{5}{|l|}{ Age } \\
\hline$\leq 50$ & $4(13.3 \%)$ & $26(86.7 \%)$ & 30 & 1 \\
\hline$>50$ & $2(10 \%)$ & $18(90 \%)$ & 20 & \\
\hline \multicolumn{5}{|l|}{ Menopausal status } \\
\hline Post & $3(10 \%)$ & $27(90 \%)$ & 30 & 0.672 \\
\hline Pre & $3(15 \%)$ & $17(85 \%)$ & 20 & \\
\hline \multicolumn{5}{|l|}{ Family history } \\
\hline Negative & $6(13.6 \%)$ & $38(86.4 \%)$ & 44 & 0.335 \\
\hline Positive & $0(0 \%)$ & $6(100 \%)$ & 6 & \\
\hline \multicolumn{5}{|l|}{ Tumor size } \\
\hline$\leq 5 \mathrm{~cm}$ & $4(9.1 \%)$ & $40(90.9 \%)$ & 44 & 0.086 \\
\hline$>5 \mathrm{~cm}$ & $2(33.3 \%)$ & $4(66.7 \%)$ & 6 & \\
\hline \multicolumn{5}{|c|}{ Lymph node positivity } \\
\hline No & $2(11.1 \%)$ & $16(88.9 \%)$ & 18 & 1 \\
\hline Yes & $4(12.5 \%)$ & $28(87.5 \%)$ & 32 & \\
\hline \multicolumn{5}{|l|}{ Disease stage } \\
\hline I \&II & $3(9.1 \%)$ & $30(90.9 \%)$ & 33 & 0.378 \\
\hline III & $3(17.6 \%)$ & $14(82.4 \%)$ & 17 & \\
\hline \multicolumn{5}{|l|}{ Tumor grade } \\
\hline GI\&GII & $4(12.1 \%)$ & $29(87.9 \%)$ & 33 & 1 \\
\hline GIII & $2(11.8 \%)$ & $15(88.2 \%)$ & 17 & \\
\hline \multicolumn{5}{|l|}{ TOP2A gene status } \\
\hline Normal & $6(14.6 \%)$ & $35(85.4 \%)$ & 41 & 0.576 \\
\hline \multicolumn{5}{|l|}{ TOP2A gene status } \\
\hline Abnormal & $0(0 \%)$ & $9(100 \%)$ & 9 & \\
\hline Amplified & $0(0 \%)$ & $5(100 \%)$ & 5 & 0.511 \\
\hline Deleted & $0(0 \%)$ & $4(100 \%)$ & 4 & \\
\hline Normal & $6(14.6 \%)$ & $35(85.4 \%)$ & 41 & \\
\hline \multicolumn{5}{|c|}{ Chromosome 17 CEP status } \\
\hline Normal & $6(14 \%)$ & $37(86 \%)$ & 43 & 0.292 \\
\hline Polysomy & $0(0 \%)$ & $7(100 \%)$ & 7 & \\
\hline \multicolumn{5}{|l|}{ Disease outcome } \\
\hline Good & $5(12.8 \%)$ & $34(87.2 \%)$ & 39 & 1 \\
\hline Poor & $1(9.1 \%)$ & $10(90.9 \%)$ & 11 & \\
\hline
\end{tabular}

parameters except with menopausal status as all cases with polysomy 17 are postmenopausal [Table 3]. There was highly statistical significant relation between TOP2A abnormalities and CEP17 polysomy as 6 out of 7 cases with chromosome 17 polysomy also showed amplification or deletion of TOP2A (Table 4).

Overall Survival (OS) and its relation to the prognostic factors

There is a statistically significant relation between OS and non-locally advanced status of the disease and radiotherapy treatment, while a trend was observed for better OS in younger age group, higher tumor grade, negative lymph nodes, low Ki-67LI, CEP17 polysomy andTOP2A alterations, with a persistent survival preference noted on cumulative survival at 12, 36 and 60 months (Table 5).

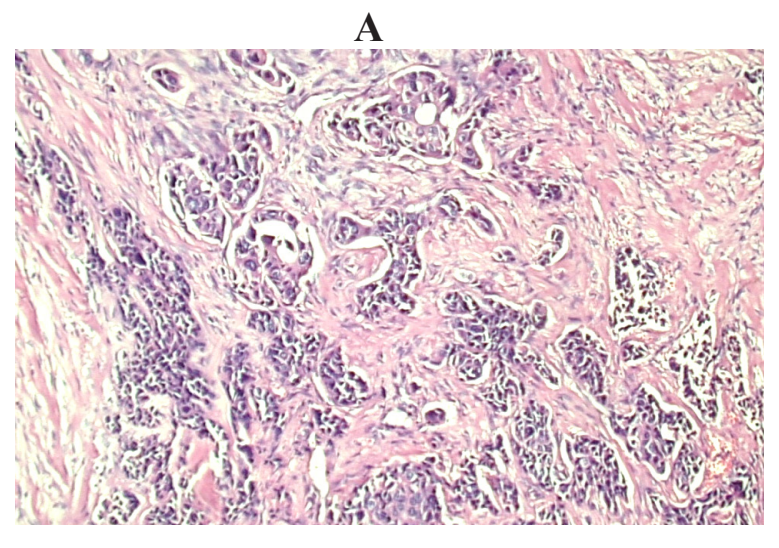

B

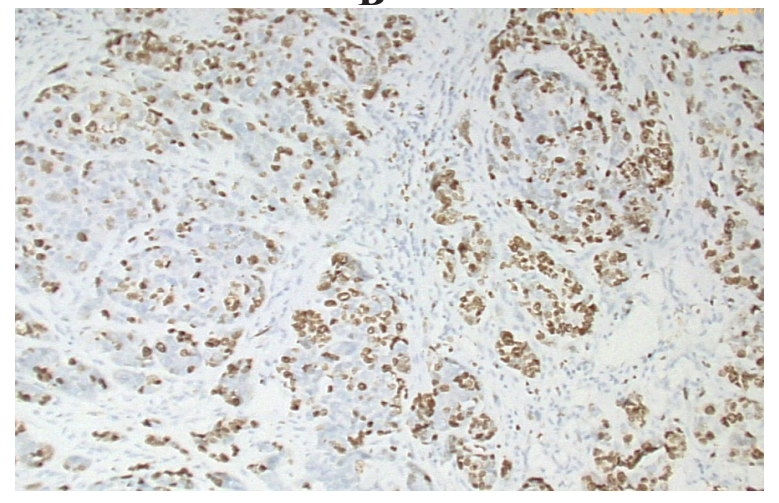

C

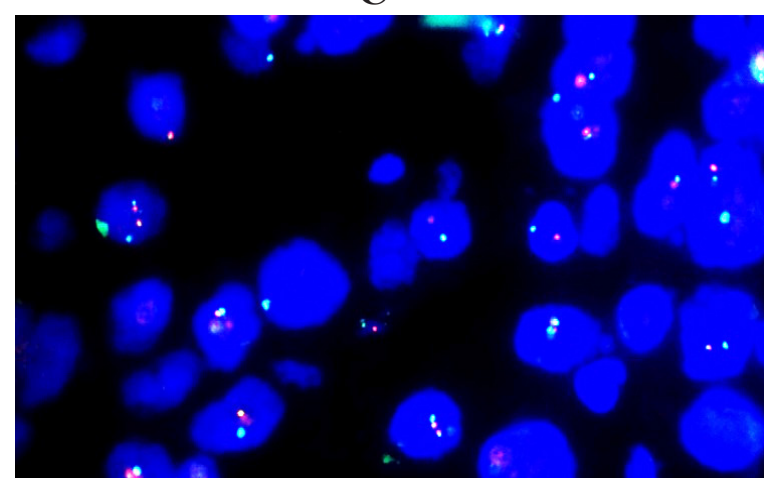

Figure 2. Invasive Duct Carcinoma, Grade II, Showing Normal CEP 17 and Normal TOP2A Gene Copy Number (A), Histologic section of the tumor, Hx and E stained (X100); (B), Immunostained slide showing high KI-67 LI about 70\%(X100). (C), Representative FISH image, showing normal chromosome 17 CEP number (as demonstrated by 1-2 aqua signals per nucleus) and normal TOP 2A gene copy number (as demonstrated by 1-2 red signals per nucleus). Gene to chromosome ratio is 1 .

Event free survival (EFS) and its relation to the prognostic factors

Twelve cases $(24 \%)$, had disease related events of either local recurrence, metastasis or death. There is a statistically significant relation between EFS and tumor higher grades, while a trend was observed for better EFS for larger tumor size, radiation therapy and the combined anthracyclines/taxanes regimens with persistent survival preference noted on cumulative survival at 12, 36 and 60 months (Table 5). 
Table 3. Relation of TOP2A Gene Status and CEP 17 with Clinicopathologic Parameters

\begin{tabular}{|c|c|c|c|c|c|c|c|}
\hline \multirow{2}{*}{$\begin{array}{l}\text { Clinicopathological } \\
\text { variable/ Category }\end{array}$} & \multirow{2}{*}{$\begin{array}{l}\text { TOP2A amplified } \\
\qquad(\mathrm{n}=5)\end{array}$} & TOP2A & \multicolumn{2}{|l|}{ TOP2A } & CEP17 & \multicolumn{2}{|l|}{ CEP17 } \\
\hline & & deleted & normal & $P$ value & Normal diploid $n=43$ & Polysomy & $\mathrm{P}$ value \\
\hline Age (years) & & $(n=4)$ & $(n=41)$ & & & $\mathrm{n}=7$ & \\
\hline$\leq 50$ & $2(6.7 \%)$ & $2(6.7 \%)$ & $26(86.7 \%)$ & & $28(93.3 \%)$ & $2(6.7 \%)$ & 0.1 \\
\hline$>50$ & $3(15 \%)$ & $2(10 \%)$ & $15(75 \%)$ & 0.47 & $15(75 \%)$ & $5(25 \%)$ & \\
\hline \multicolumn{8}{|l|}{ Menopausal status } \\
\hline Post & $3(10 \%)$ & $4(13.3 \%)$ & $23(76.7 \%)$ & & $23(76.7 \%)$ & $7(23.3 \%)$ & 0.033 \\
\hline Pre & $2(10 \%)$ & $0(0 \%)$ & $18(90 \%)$ & 0.292 & $20(100 \%)$ & $0(0 \%)$ & \\
\hline \multicolumn{8}{|l|}{ Family history } \\
\hline Negative & $5(11.4 \%)$ & $4(9.1 \%)$ & $35(79.5 \%)$ & 1 & $37(84.1 \%)$ & $7(15.9 \%)$ & 0.292 \\
\hline Positive & $0(0 \%)$ & $0(0 \%)$ & $6(100 \%)$ & & $6(100 \%)$ & $0(0 \%)$ & \\
\hline \multicolumn{8}{|l|}{ Tumor size } \\
\hline$\leq 5 \mathrm{~cm}$ & $4(9.1 \%)$ & $4(9.1 \%)$ & $36(81.8 \%)$ & 0.717 & $37(84.1 \%)$ & $7(15.9 \%)$ & 0.292 \\
\hline$>5 \mathrm{~cm}$ & $1(16.7 \%)$ & $0(0 \%)$ & $5(83.3 \%)$ & & $6(100 \%)$ & $0(0 \%)$ & \\
\hline \multicolumn{8}{|c|}{ Lymph node positivity } \\
\hline Yes & $3(9.4 \%)$ & $3(9.4 \%)$ & $26(81.3 \%)$ & 1 & $16(88.9 \%)$ & $2(11.1 \%)$ & 1 \\
\hline No & $2(11.1 \%)$ & $1(5.6 \%)$ & $15(83.3 \%)$ & & $27(84.4 \%)$ & $5(15.6 \%)$ & \\
\hline \multicolumn{8}{|l|}{ Disease stage } \\
\hline I\&II & $4(12.1 \%)$ & $2(6.1 \%)$ & $27(81.8 \%)$ & $*$ & $28(84.8 \%)$ & $5(15.2 \%)$ & 0.744 \\
\hline III & $1(5.9 \%)$ & $2(11.8 \%)$ & $14(82.4 \%)$ & & $15(88.2 \%)$ & $2(11.8 \%)$ & \\
\hline \multicolumn{8}{|l|}{ Tumor grade } \\
\hline G I\&GII & $3(9.1 \%)$ & $3(9.1 \%)$ & $27(81.8 \%)$ & 1 & $27(81.8 \%)$ & $6(18.2 \%)$ & 0.398 \\
\hline G III & $2(11.8 \%)$ & $1(5.9 \%)$ & $14(82.4 \%)$ & & $16(94.1 \%)$ & $1(5.9 \%)$ & \\
\hline \multicolumn{8}{|l|}{ Ki-67 LI } \\
\hline$\leq 20 \%$ & $0(0 \%)$ & $0(0 \%)$ & $6(100 \%)$ & 0.511 & $6(100 \%)$ & $0(0 \%)$ & 1 \\
\hline$>20 \%$ & $5(11.4 \%)$ & $4(9.1 \%)$ & $35(79.5 \%)$ & & $37(84.1 \%)$ & $7(15.9 \%)$ & \\
\hline \multicolumn{8}{|l|}{ Disease outcome } \\
\hline Good & $4(10.3 \%)$ & $4(10.3 \%)$ & $31(79.5 \%)$ & 0.812 & $32(82.1 \%)$ & $7(17.9 \%)$ & 0.13 \\
\hline Poor & $1(9.1 \%)$ & $0(0 \%)$ & $10(90.9 \%)$ & & $11(100 \%)$ & $0(0 \%)$ & \\
\hline
\end{tabular}

*P value cannot be calculated due to small number within stratum.

A

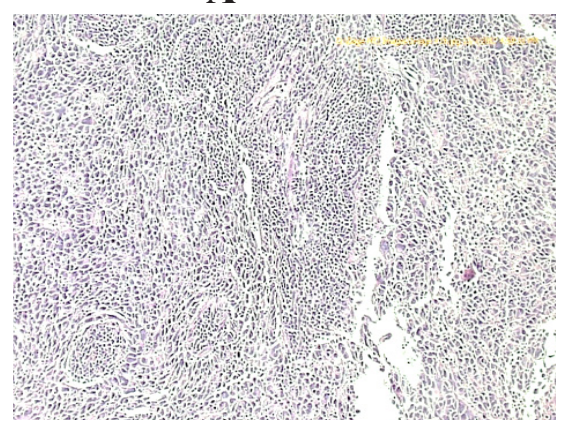

B

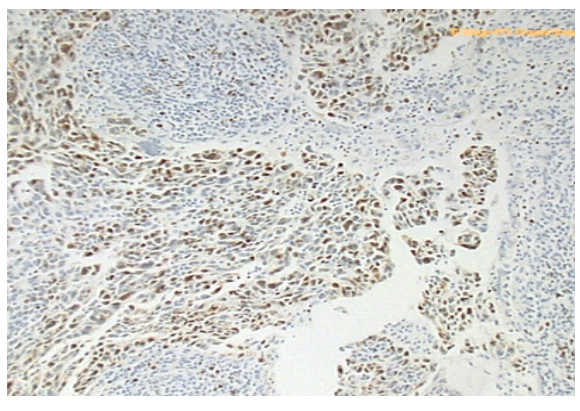

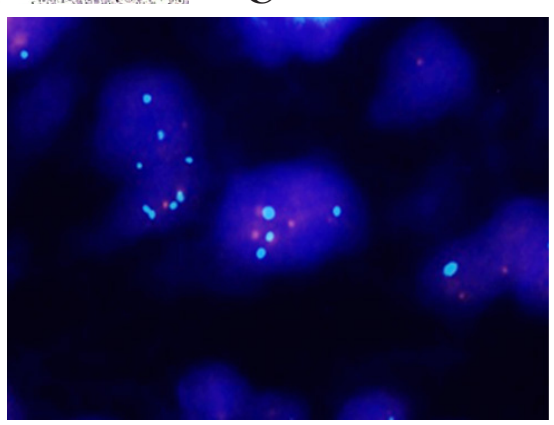

Figure 3. Invasive Duct Carcinoma, Grade III, with CEP 17 Polysomy and TOP2A Gene Deletion (A), Histologic section of the tumor (X 40); (B), Immunostained slide for KI-67 LI showing proliferation rate 80\% (X 40); (C), Representative FISH image showing CEP 17 polysomy (as demonstrated by 4-5 aqua signals) and TOP2A gene deletion (as demonstrated by 2-3 red signals). Gene to chromosome ratio is $<0.8$. 
Table 4. Relation between TOP2A Gene Status and CEP 17

\begin{tabular}{llccccc}
\hline TOP2A status & $\begin{array}{c}\text { TOP2A } \\
\text { Amplified } \\
(\mathrm{n}=5)\end{array}$ & $\begin{array}{c}\text { TOP2A } \\
\text { Deleted } \\
(\mathrm{n}=4)\end{array}$ & $\begin{array}{c}\text { TOP2A } \\
\text { Normal } \\
(\mathrm{n}=41)\end{array}$ & Total & P value \\
\hline Chromosome 17CEP & Normal & $3(7 \%)$ & $0(0 \%)$ & $40(93 \%)$ & $43(100 \%)$ & $<0.001$ \\
& Polysomy & $2(28.6 \%)$ & $4(57.1 \%)$ & $1(14.3 \%)$ & $7(100 \%)$ & $<$ \\
\hline
\end{tabular}

\section{Discussion}

In our study, TOP2A gene alterations were detected in $9 / 50$ cases; $18 \%$ (amplification in 5 cases; $10 \%$ and deletion in 4 cases; $8 \%$ ). These data were not similar to

A

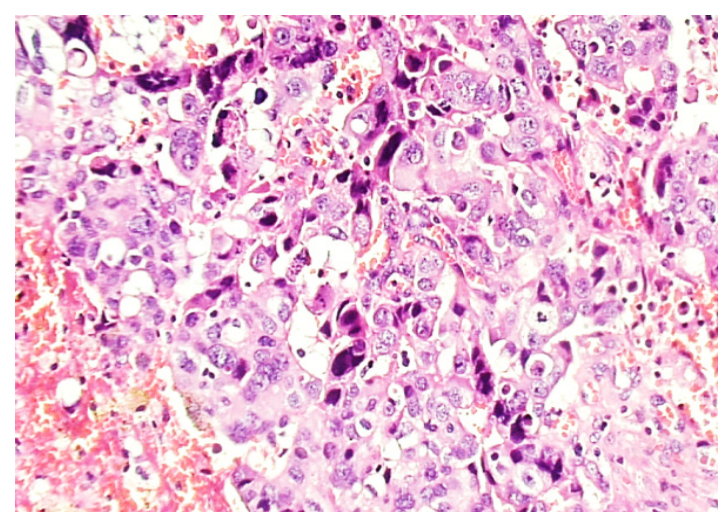

C percentages recorded in literature about TNBC, as in a study by Almeida et al., (2014), TOP2A gene alteration was seen in $7.1 \%$ by SISH technique. Fawzi and Alqanbar, (2017) detected TOP2A gene amplification in 3.6\% of cases by CISH technique. Fountzilas et al., (2013),

\section{B}

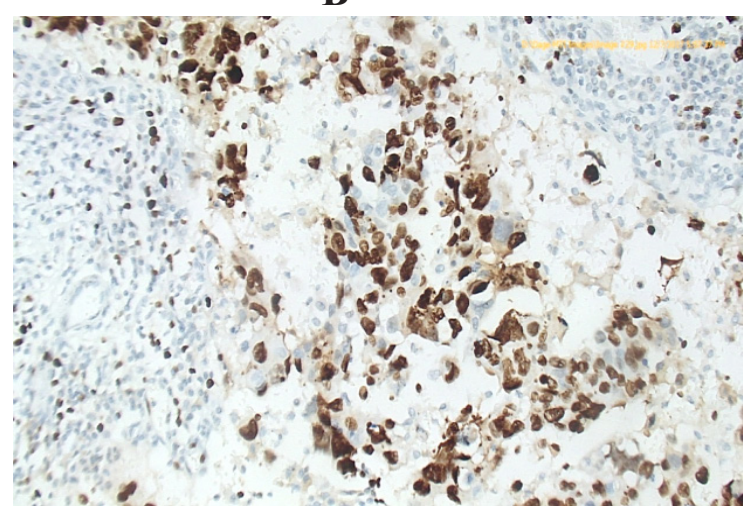

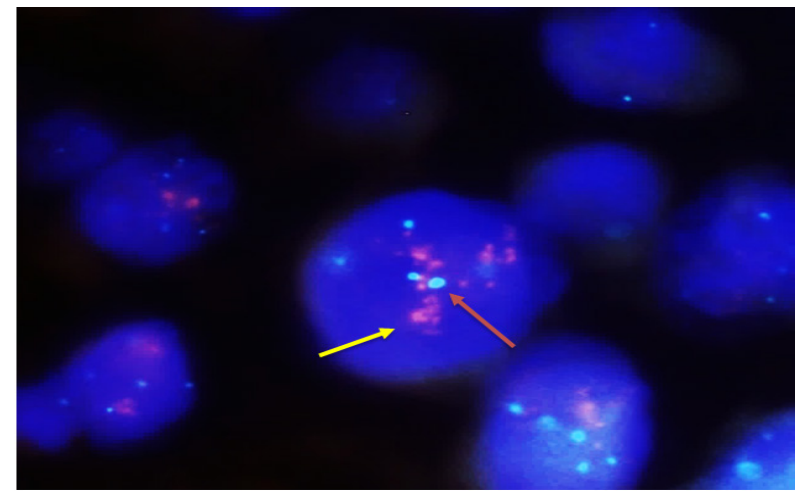

Figure 4. Invasive Duct Carcinoma, Grade III with CEP 17 Polysomy and Amplified TOP2A Gene Copy Number. (A), Histologic section of the tumor, Hx\&E stained (X200); (B), Immunostained slide for KI-67 LI, showing high proliferation rate $90 \%,(x 200)$. (C), Representative FISH image, showing CEP17 polysomy and amplified TOP2A gene copy number (as demonstrated by 4-5 aqua signals for the chromosome "red arrows" \& red clusters for the gene denoting high amplification pattern "yellow arrows"). Gene to chromosome ratio is $>2$.

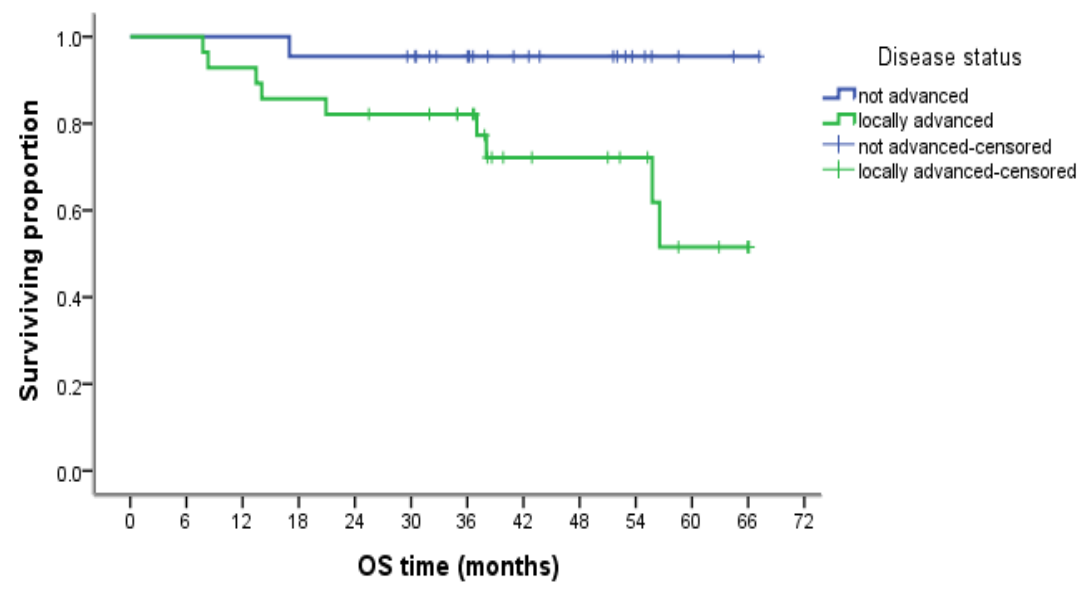

Figure 5. Kaplan-Meier Analysis of Overall Survival According to Disease Status

3586 Asian Pacific Journal of Cancer Prevention, Vol 19 
Table 5. Overall Survival (OS) and Event Free Survival(EFS) and Their Relations to the Prognostic Factors

\begin{tabular}{|c|c|c|c|c|c|}
\hline \multirow[b]{2}{*}{$\begin{array}{l}\text { Clinicopathological } \\
\text { variables in Whole } \\
\text { group }\end{array}$} & \multirow[b]{2}{*}{ No. } & \multicolumn{2}{|c|}{ OS } & \multicolumn{2}{|c|}{ EFS } \\
\hline & & $\begin{array}{l}\text { No. of } \\
\text { events }\end{array}$ & $\begin{array}{c}\mathrm{p}- \\
\text { value }\end{array}$ & $\begin{array}{l}\text { No.of } \\
\text { events }\end{array}$ & $\begin{array}{c}\mathrm{P}- \\
\text { value }\end{array}$ \\
\hline & 50 & 10 & - & 12 & - \\
\hline \multicolumn{6}{|l|}{ Age groups } \\
\hline$\leq 50$ years & 30 & 4 & 0.184 & 6 & 0.536 \\
\hline$>50$ years & 20 & 6 & & 6 & \\
\hline \multicolumn{6}{|l|}{ Menopausal status } \\
\hline Post & 30 & 7 & 0.608 & 9 & 0.326 \\
\hline Pre & 20 & 3 & & 3 & \\
\hline \multicolumn{6}{|l|}{ Family history } \\
\hline Negative & 44 & 8 & 0.484 & 10 & 0.471 \\
\hline Positive & 6 & 2 & & 2 & \\
\hline \multicolumn{6}{|l|}{ Tumor size } \\
\hline$\leq 5 \mathrm{~cm}(\mathrm{~T} 1 \& \mathrm{~T} 2)$ & 44 & 9 & 0.727 & 12 & 0.185 \\
\hline$>5 \mathrm{~cm}(\mathrm{~T} 3 \& \mathrm{~T} 4)$ & 6 & 1 & & 0 & \\
\hline \multicolumn{6}{|l|}{ Lymph nodes status: } \\
\hline Negative & 18 & 1 & 0.097 & 4 & 0.723 \\
\hline Positive & 32 & 9 & & 8 & \\
\hline Tumor stage & 33 & 5 & & 33 & \\
\hline I\&II & 17 & 5 & 0.222 & 17 & 0.862 \\
\hline \multicolumn{6}{|l|}{ III } \\
\hline Tumor grade & & & & 11 & 0.034 \\
\hline G1\&G2 & 33 & 9 & 0.063 & 1 & \\
\hline G3 & 17 & 1 & & & \\
\hline Ki-67 LI & & & & 1 & 0.586 \\
\hline$\geq 20 \%$ & 44 & 10 & 0.148 & 11 & \\
\hline$<20 \%$ & 6 & 0 & & & \\
\hline \multicolumn{6}{|l|}{ Radiotherapy } \\
\hline No & 26 & 8 & 0.03 & 8 & 0.135 \\
\hline yes & 24 & 2 & & 4 & \\
\hline \multicolumn{6}{|l|}{ Chemotherapy } \\
\hline $\begin{array}{l}\text { Anthracycline \& } \\
\text { Taxane }\end{array}$ & 36 & 5 & 0.212 & 6 & 0.104 \\
\hline Anthracycline only & 14 & 5 & & 6 & \\
\hline TOP2A gene status: & & & & 1 & \\
\hline Amplified & 5 & 1 & & 1 & 0.984 \\
\hline Deleted & 4 & 0 & $*$ & 10 & \\
\hline Normal & 41 & 9 & & & \\
\hline TOP2A gene status & & & & 10 & 0.873 \\
\hline Normal & 41 & 9 & 0.524 & 2 & \\
\hline Abnormal & 9 & 1 & & & \\
\hline CEP 17 chromosome: & & & & 11 & 0.456 \\
\hline Normal & 43 & 10 & 0.193 & 1 & \\
\hline Polysomy & 7 & 0 & & & \\
\hline Disease status: & & & & & 0.729 \\
\hline Not advanced & 22 & 1 & 0.034 & 5 & \\
\hline Locally advanced & 28 & 9 & & 7 & \\
\hline $\begin{array}{l}\text { TOP2A\&CEP } 17 \\
\text { combined: }\end{array}$ & & & & 2 & 0.697 \\
\hline Abnormal & 10 & 1 & 0.369 & 10 & \\
\hline Normal & 40 & 9 & & & \\
\hline
\end{tabular}

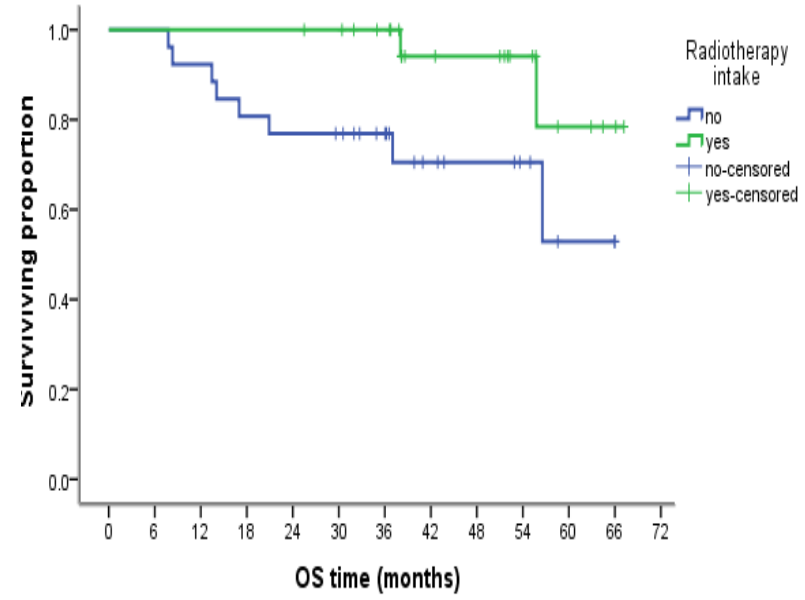

Figure 6. Kaplan-Meier Analysis of Overall Survival According to Radiation Therapy

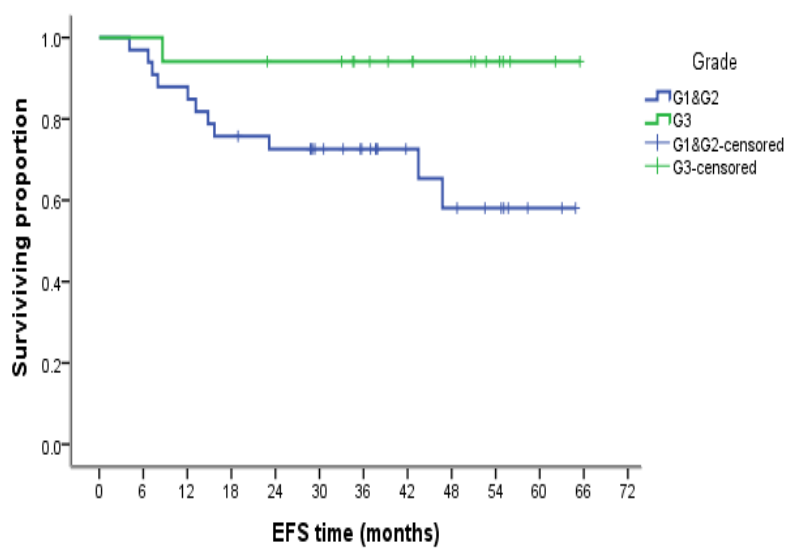

Figure 7 . Kaplan-Meier Analysis of Event Free Survival According to Tumor Grade

detected TOP2A gene deletion by FISH technique in $6.3 \%$ of cases. Murria et al., (2015) had noted by the usage of multiple ligation probes amplification (MLPA) technique; amplification of TOP $2 \mathrm{~A}$ gene in $4 \%$ and deletion in $4 \%$ of their TNBC patients. These differences in the percentage of gene copy number alterations may be explained by the different techniques and cutoff values used to estimate the gene copy number. Yet, these results may also point to different genetic alterations involved in the pathogenesis of cancer according to study population and their demographic and ethnic background with observed higher TOP2A alterations among Egyptian TNBC cases.

TOP2A gene amplification was absent in all cases with positive family history $(0 / 6)$. In a study by El-Gerzawy et al., (2013), TOP2A gene amplification was observed in two cases with positive family history $(16.7 \%)$ but not observed in cases lacking positive family history.

Longer EFS was noticed in patients with TOP2A alterations compared to those with normal TOP2A, this was consistent with results reported by (Almeida et al., 2014). Li et al., (2016), had also reported an improved disease free survival and OS in cases with TOP2A gene amplification and this was in alignment with most of the published data on TOP2A in literature. This could be explained by that patients with TOP2A alterations 
were those who benefited most from the anthracycline based chemotherapy which was reflected on the observed longer EFS among our cases. This point is of paramount importance as it highlights the significance of estimation of TOP2A status as a strong prognostic and predictor factor for response to anthracycline based chemotherapy, the same was proved by (Knoop et al., 2005; O’Malley et al., 2009).

CEP 17 polysomy was detected in $14 \%$ of cases $(n=7)$, this was not so near from results of (Fountzilas et al., 2013), (26.2\%) on their study on TNBC cases. In our study, CEP 17 polysomy showed a non-statistical trend towards ages older than 50 years, $(p=0.10)$. It was observed only in postmenopausal patients, $(\mathrm{P}=0.03)$, similar to what noticed by Fountzilas et al., (2013). CEP 17 polysomy was only noticed in tumors $\leq 5 \mathrm{~cm}$, but not in tumors $>5 \mathrm{~cm}$. It was also more observed in low grade tumors. In their studies, (Krishnamurti et al., 2009; Fountzilas et al., 2013), and contrary to ours, there was a significant correlation with high grade tumors, while Salido et al., (2005) showed no significant correlation of CEP17 polysomy with tumor grade. CEP17 polysomy had a significant correlation with TOP2A alterations, $p$ value $<0.001$. This was in line with a study done by (Fountzilas et al., 2013).

In our study, Ki-67 LI was higher within higher grade tumors; $70.6 \%$ of grade III cases. This was consistent with the studies done by Hao et al., (2016) and Qiu et al., (2016).

We observed that all cases with TOP2A alterations and all cases with CEP17 polysomy are of high Ki-67 LI, yet this relation did not reach point of statistical significance, mostly due to the small sample size; these results are expected being both as markers of proliferation. In concordance with our study, Mrklić et al., (2014), observed a strong association of amplified TOP2A with Ki-67 LI. Additionally, Petroni et al., (2012), noted a strong association between high $\mathrm{Ki}-67 \mathrm{LI}$ and the polysomic status.

Radiotherapy showed a statistically significant better OS, this was also proved by (Chen et al., 2013; Gado, et al., 2016) and points to the importance of multimodality approach in treatment of this aggressive molecular subtype of breast cancer. There was a non-statistical trend towards longer EFS noted in patients who received adjuvant radiotherapy $(p=0.135)$, this was in consistence with results obtained by (Chen et al., 2013).

In our study, there was a better OS in patients group treated by combined anthracyclines and taxanes compared to those treated by anthracyclines alone $82.2 \%$ and $47.12 \%$, this was in concordance with what reported by (Rodler et al., 2011; Gado et al., 2016). EFS was longer with patients treated by combined anthracyclines and taxanes versus than those who were treated by anthracyclines only 83.1 $\%$ and $53.6 \%$, respectively, although $\mathrm{P}$ value was not significant (0.104).

Metastasis was seen in 6/50 cases and recurrence was reported in $4 / 50$ cases. Forty out of fifty cases are still alive, while 5/50 cases had died, 5/50 cases showed lost follow up data. This observation of good outcome of our study population may be not expected as regard the aggressive nature of TNBC. Yet with the relatively newly addressed treatment of the Anthracycline/Taxane based chemotherapy given to our patients in NCI, it is obvious that this line of treatment was effective. Still, longer follow up of the patients is definitively needed to monitor long term efficacy and validity of this treatment.

Univariate analysis for EFS, showed that only high tumor grade had significant effect. The better prognosis noted in higher grades may be a reflection to the better response to chemotherapy noted in higher grade tumors than in lower grades, this was in concordance with the study done by Pinder et al., (1998). EFS was better in combined chemotherapy regimens, radiation therapy and larger tumor sizes, however without statistical significance.

In view of our results, we conclude the importance of selecting the appropriate candidates of TNBC to adjuvant anthracycline based chemotherapy. Selection must be done on a basis of potentially approved biological predictors such as FISH technique for detection of TOP2A gene copy number alterations (amplification or deletion) and for detection of chromosome $17 \mathrm{CEP}$ status. Our results pointed to an association between TOP2A alterations and CEP 17 polysomy which was reflected on more responsiveness to chemotherapy and so better disease outcome.

We also concluded the importance of applying taxanes to the chemotherapy regimen and the adjuvant radiotherapy in the treatment plan, based on our observations about the better prognosis noted in patients treated with them.

\section{References}

Almeida D, Gerhard R, Leitão D, et al (2014). Topoisomerase IIalfa gene as a predictive marker of response to anthracyclines in breast cancer. Pathol Res Pract, 210, 675-9.

Arriola E, Marchio C, Tan DS, et al (2008). Genomic analysis of the HER2/TOP2A amplicon in breast cancer and breast cancer cell lines. Lab Invest, 88, 491-503.

Ballinger T, Kremer J, Miller K (2016).Triple negative breast cancer-Review of current and emerging therapeutic strategies. Oncol Hematol Rev, 12, 89-94.

Brown DC, Gatter KC(2002). Ki67 protein: the immaculate deception?. Histopathology, 40, 2-11.

Chen X, Yu X, Chen J ,et al (2013). Radiotherapy can improve the disease-free survival rate in triple-negative breast cancer patients with T1-T2 disease and one to three positive lymph nodes after mastectomy. Oncologist, 18, 141-7.

Edge SB, Compton CC (2010). The American joint committee on cancer: the 7th edition of the AJCC cancer staging manual and the future of TNM. Ann Surg Oncol, 17, 1471-4.

El-Gerzawy AMS, Al Ansary MMS , Hussein IL, et al (2013). Study of Her-2/neu and TOP2A expression in familial versus sporadic breast carcinoma. Med Res J, 12, 21-9.

Elston CW, Ellis IO (1991). Pathological prognostic factors in breast cancer. I. The value of histological grade in breast cancer: experience from a large study with long-term follow-up. Histopathology, 19, 403-10.

Fawzi M, Alqanbar A (2017). Role of topoisomerase II alpha gene status on selection of anthracycline therapy in triple negative breast cancer. Med J Babylon, 14, 189-97.

Fountzilas G, Dafni U, Bobos M, et al (2013). Evaluation of the prognostic role of centromere 17 gain and HER2/ topoisomerase II alpha gene status and protein expression in patients with breast cancer treated with anthracycline- 
containing adjuvant chemotherapy: Pooled analysis of two Hellenic Cooperat. BMC Cancer, 13, 163.

Fritz P, Cabrera CM, Dippon J, et al (2005). c-erbB2 and topoisomerase II $\alpha$ protein expression independently predict poor survival in primary human breast cancer: A retrospective study. Breast Cancer Res, 7, R374-84.

Gado N, Ibrahim D, Atef D, Kanaan A (2016). Clinical characteristics of triple negative breast cancer in Egyptian women: a hospital-based experience. Cancer Ther Oncol Int $J, \mathbf{4}, 426$.

Gerdes J, Schwab U, Lemke H, Stein H (1983). Production of a mouse monoclonal antibody reactive with a human nuclear antigen associated with cell proliferation. Int J Cancer, $\mathbf{3 1}$, 13-20.

Gianni L, Norton L, Wolmark Ng et al (2009). Role of anthracyclines in the treatment of early breast cancer. J Clin Oncol, 27, 4798-808.

Greenberg RA (2008). Recognition of DNA double strand breaks by the BRCA1 tumor suppressor network. Chromosoma, 117, 305-17.

Hao S , He Z, Yu K, Yang Wand Shao Z (2016). New insights into the prognostic value of Ki-67 labeling index in patients with triple-negative breast cancer. Oncotarget, 7.

Jacot W, Fiche M, Zaman K, Wolfer Aand Lamy PJ (2013). The HER2 amplicon in breast cancer: Topoisomerase IIA and beyond. Biochimica et Biophysica Acta - Reviews on Cancer. Elsevier, 1836, pp 146-57.

Kellner U, Sehested M, Jensen PB, Gieseler F, Rudolph P (2002). Culprit and victim - DNA topoisomerase II. Lancet Oncol, 3, 235-43.

Knoop AS, Knudsen H, Balslev E, et al (2005). retrospective analysis of topoisomerase IIa amplifications and deletions as predictive markers in primary breast cancer patients randomly assigned to cyclophosphamide, methotrexate, and fluorouracil or cyclophosphamide, epirubicin, and fluorouracil: Danish Breast Cancer Cooperative Group. Am Soc Clin Oncol, 23, 7483-90.

Krishnamurti U, Hammers JL, Atem FD, Storto PD, Silverman JF (2009). Poor prognostic significance of unamplified chromosome 17 polysomy in invasive breast carcinoma. Mod Pathol, 22, 1044-8.

Leo ADi, Desmedt C, Bartlett JMS, et al (2011). HER2 and TOP2A as predictive markers for anthracycline-containing chemotherapy regimens as adjuvant treatment of breast cancer: a meta-analysis of individual patient data. Lancet Oncol, 12, 1134-42.

Li XY, Li M, Jin F (2016). Topoisomerase II alpha and BRCA1 expression as predictive factors for anthracyclinebased adjuvant chemotherapy response and prognosis in triple-negative breast cancers. Int J Clin Exp Pathol, 9, 9249-58.

Mitrović O, Čokić V, Đikić D, et al (2014). Correlation between ER, PR, HER-2, Bcl-2, p53, proliferative and apoptotic indexes with HER-2 gene amplification and TOP2A gene amplification and deletion in four molecular subtypes of breast cancer. Target Oncol, 9, 367-79.

Mrklić I, Pogorelić Z, Ćapkun Vand Tomić S (2014). Expression of topoisomerase II- $\alpha$ in triple negative breast cancer. Appl Immunohistochem Mol Morphol, 22, 182-7.

Murria R, Palanca S, de Juan I, et al (2015). Immunohistochemical, genetic and epigenetic profiles of hereditary and triple negative breast cancers. Relevance in personalized medicine. Am J Cancer Res, 5, 2330-43.

Nishimura R, Osako T, Nishiyama Y, et al (2014). Prognostic significance of Ki-67 index value at the primary breast tumor in recurrent breast cancer. Mol Clin Oncol, 2, 1062-8.

Nishimura R, Osako T, Okumura Y, et al (2010). Ki-67 as a prognostic marker according to breast cancer subtype and a predictor of recurrence time in primary breast cancer. Exp Therap Med, 1, 747-54.

O’Malley FP, Chia S, Tu D, et al (2009). Topoisomerase II alpha and responsiveness of breast cancer to adjuvant chemotherapy. J Natl Cancer Inst, 101, 644-50.

Olivier M, Petitjean A, Marcel V, et al (2009). Recent advances in p53 research: an interdisciplinary perspective. Cancer Gene Ther, 16, 1-12.

Olsen KE, Knudsen H, Rasmussen BB, et al (2004). Amplification of HER2 and TOP2A and deletion of TOP2A genes in breast cancer investigated by new FISH probes. Acta Oncol, 43, $35-42$.

Perez, EA, Reinholz MM, Hillman DW, et al (2010). HER2 and chromosome 17 effect on patient outcome in the N9831 adjuvant trastuzumab trial. Am Soc Clin Oncol, 28, 4307-15.

Petroni S, Addati T, Mattioli E, et al (2012). Centromere 17 copy number alteration: Negative prognostic factor in invasive breast cancer?. Arch Pathol Lab Med, 136, 993-1000.

Pinder SE, Murray S, Ellis IO, et al (1998). The importance of the histologic grade of invasive breast carcinoma and response to chemotherapy. Cancer, 83, 1529-39.

Qiu J, Xue X, Hu C, et al (2016). Comparison of clinicopathological features and prognosis in triple-negative and non-triple negative breast cancer. Cancer, 7, 167-73

Rodler E, Korde L, Gralow J (2010). Current treatment options in triple negative breast cancer. Breast Dis, 32, 99-122.

Salido M, Tusquets I, Corominas JM, et al (2005). Polysomy of chromosome 17 in breast cancer tumors showing an overexpression of ERBB2: a study of 175 cases using fluorescence in situ hybridization and immunohistochemistry. Breast Cancer Res, 7, R267-73.

Siegel RL, Miller KD, Jemal A (2018). Cancer statistics, 2018. CA Cancer J Clin, 68, 7-30.

Untch M, Gerber B, Harbeck N, et al (2013). 13th St. Gallen international breast cancer conference 2013: Primary therapy of early breast cancer evidence, controversies, consensus - Opinion of a German team of experts (Zurich 2013). Breast Care, 8, 221-9.

Yamamoto S, Ibusuki M,Yamamoto Y, et al (2013). Clinical relevance of $\mathrm{Ki} 67$ gene expression analysis using formalin-fixed paraffin-embedded breast cancer specimens. Breast Cancer, 20, 262-70.

Yao H, He G, Yan S, et al (2015). Triple-negative breast cancer: is there a treatment on the horizon?. Oncotarget, 8, 1913-24.

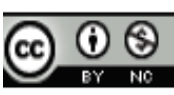

This work is licensed under a Creative Commons AttributionNon Commercial 4.0 International License. 